\title{
MANIFOLDS OF ALMOST HALF OF THE MAXIMAL VOLUME
}

\author{
OGUZ C. DURUMERIC
}

(Communicated by David G. Ebin)

\begin{abstract}
The Riemannian manifolds with sectional curvature $\geq 1$ and volume slightly less (in terms of the dimension and the upper bound of the sectional curvature) than $V$, the half of the volume of the standard sphere, are classified. The behavior of the critical points of the distance function on manifolds whose volume differ from $V$ in terms of constructible constants in terms of the dimension is discussed.
\end{abstract}

1. Introduction. Let $\left(M^{n}, g\right)$ be a compact smooth Riemannian manifold, and $K(M, g), d(M, g), v(M, g)$ denote its sectional curvature, diameter and volume. We study the manifolds with positive sectional curvature, and normalize the metric so that $K(M, g) \geq 1$, throughout this paper when $g$ is smooth. Let $v\left(S^{n}, c a n\right)=2 V_{0}$.

By the work of Grove-Shiohama [GS] and Gromoll and Grove [GG1-GG4]: if $d(M, g)>\pi / 2$ then $M$ is homeomorphic to a sphere, and if $d(M, g)=\pi / 2$ then either (i) $M$ is homeomorphic to a sphere, or (ii) $(M, g)$ is isometric to $\mathbf{C P}^{k}$ or $\mathbf{H P}^{s}$ with their canonical metrics, or (iii) $\pi_{1}(M)=1$ and $H^{*}(M, \mathbf{Z})=H^{*}\left(\mathbf{C a P}^{2}, \mathbf{Z}\right)$ or, (iv) $M$ is not simply-connected and its universal cover is isometric to $\left(S^{n}\right.$, can $)$ or $\left(\mathbf{C P}^{k}\right.$, can) with $k=n / 2$ odd.

By volume comparison, Bishop and Crittenden [BC], if $v(M, g) \geq V_{0}$, then $d(M, g) \geq \pi / 2$. However, if $v(M, g) \geq V_{0}$, one obtains that $M$ is homeomorphic to a sphere, or isometric to $\left(\mathbf{R P}^{n}\right.$, can) which occurs only when $v(M, g)=V_{0}$ and $d(M, g)=\pi / 2$.

Under the condition $K \geq K(M, g) \geq 1$, the manifolds with $v(M, g)>V_{0}-c$ is a subset of the set of the manifolds with $d(M, g)>(\pi / 2)-c^{\prime}$ which were classified in [D] to be homeomorphic to a sphere, to have the cohomology ring of (or with a slightly stronger hypothesis, diffeomorphic to) $\mathbf{C P}^{k}, \mathbf{H P}^{s}$, or $\mathbf{C a \mathbf { P } ^ { 2 }}$, or to be diffeomorphic to a non-simply-connected manifold whose universal cover is isometric to $\left(S^{n}\right.$, can $)$ or $\left(\mathbf{C P}^{k}\right.$, can $)$. The proof of this basically involves taking a sequence of metrics $\left(M, g_{k}\right)$ with $d\left(M, g_{k}\right)$ converging to $\pi / 2$, extracting a convergent subsequence in the sense of Hausdorff-Lipschitz by Gromov's Compactness Theorem $[\mathbf{G v}, \mathbf{P}]$, converging to $\left(M, g_{0}\right)$ which is $C^{1, \alpha}$ a priori, $\alpha<1$, then give a proof similar to [GG3] for $C^{1, \alpha}$ metric $g_{0}$ with $d\left(M, g_{0}\right)=\pi / 2$. Hence it is natural to expect that the following is true.

THEOREM 1. Let $\left(M, g_{k}\right)$ be a sequence of smooth Riemannian metrics with $1 \leq K\left(M, g_{k}\right) \leq K, d\left(M, g_{k}\right)$ converging to $\pi / 2$ and $v\left(M, g_{k}\right)$ converging to $V_{0}$

Received by the editors March 25, 1987 and, in revised form, October 2, 1987.

1980 Mathematics Subject Classification (1985 Revision). Primary 53C20.

Key words and phrases. Curvature, volume, diameter.

Research was partially supported by NSF grant DMS85-04984.

(C) 1988 American Mathematical Society $0002-9939 / 88 \$ 1.00+\$ .25$ per page 
$\left(=v\left(\mathbf{R} \mathbf{P}^{n}\right.\right.$, can $\left.)\right)$. Then there exists a subsequence converging to $\left(\mathbf{R} \mathbf{P}^{n}\right.$, can $)$ in the Hausdorff-Lipschitz sense.

COROLlARY. Given $n$ and $K_{0}$, there exist positive constants $c_{1}$ and $c_{2}$ depending on $n$ and $K_{0}$ such that for manifolds $\left(M^{n}, g\right)$ with $1 \leq K(M, g) \leq K_{0}$ one has

(a) if $v(M, g)>V_{0}-c_{1}$ then $M$ is homeomorphic to a sphere or diffeomorphic to $\mathbf{R P}^{n}$,

(b) if $v(M, g)>V_{0}-c_{1}$ and $d(M, g) \leq \pi / 2$ then $M$ is diffeomorphic to $\mathbf{R P}^{n}$, and

(c) there exists no Riemannian manifold with $v(M, g)>V_{0}-c_{1}$ and $\pi / 2<$ $d(M, g)<(\pi / 2)+c_{2}$.

Obviously, one can find $\left(M, g_{0}\right)$ as above and $d\left(M, g_{0}\right)=\pi / 2$ and $v\left(M, g_{0}\right)=V_{0}$, but one cannot immediately conclude that $\left(M, g_{0}\right)$ is isometric to $\left(\mathbf{R P}^{n}\right.$, can $)$, since $g_{0}$ is not known to be smooth. Especially, there are examples of nonsmooth limit metrics on spheres. The proof of Theorem 1 involves volume related estimates for $\left(M, g_{k}\right)$ together with some results from [D]. Even though we can compare the volumes of metric balls or any other star shaped region in $\left(M, g_{0}\right)$ to $\left(S^{n}, c a n\right)$, to prove constant sectional curvature from the maximal volume may involve Jacobi fields which may not exist for $\left(M, g_{0}\right)$. The estimates we obtain in Theorem 2 are given in terms of explicitly constructed universal constants which does not depend on the upper bound of sectional curvature. So they may be of interest alone. For the notation see $\S \S 2,3$.

In a recent work of $\mathrm{K}$. Grove and P. Petersen [GP2], a homotopy version of Corollary of Theorem 1 is obtained by a different method with a weaker hypothesis, i.e. $c_{1}$ does not depend on $K_{0}$ and its value can be calculated. Grove and Petersen also simultaneously and independently obtained Proposition 3.3 in the appendix of [GP1], which is phrased and proved in a different way.

THEOREM 2. Let $(M, g)$ be a smooth Riemannian manifold with $K(M, g) \geq 1$ and $V_{0}=v\left(\mathbf{R P}^{n}\right.$, can $)$. For any subset $A \subseteq S^{n-1}, 0 \leq x \leq y \leq \pi$, and $0 \leq z \leq \pi$ define $\lambda(A, x, y, z)$ to be the volume of the region $W(A)$ in $S^{n}$ where $p^{\prime} \in S^{n}$ is arbitrary, $U S_{p^{\prime}}^{n}$ is identified with $S^{n-1}$ isometrically and $W(A)=\bar{B}\left(z, p^{\prime}\right)$ $\bigcup_{v \in A} B\left(x, \exp _{p^{\prime}} y v\right)$. Then

(a) If $A$ spans $S^{n-1}$, then $\lambda(A, x, y, z) \leq \lambda\left(S^{0}, x, y, z\right)$, and equality holds only if $A=S^{0}$ provided that $x>0$ and $\lambda\left(S^{0}, x, y, z\right)>0$, where $S^{0}$ is any antipodal pair.

(b) $\operatorname{vol}_{n}(M-B(r, p)) \leq \lambda(L(q, p), r, d(q, p), d(M, g))$, for any $p, q \in M$, and $r \leq d(p, q)$.

(c) If $K(M, g) \geq 1, v(M, g)>V_{0}-\varepsilon, d(M, g) \leq \pi / 2, p \in M$ and $q$ is a nontrivial critical point for $d(p$,$) , then there exist explicitly constructible positive universal$ constants as follows.

(i) $d(p, q)>\pi / 2-c_{1}(n, \varepsilon)$.

(ii) For all $v_{1}, v_{2} \in L(q, p), \alpha\left(v_{1}, v_{2}\right)<\alpha_{0}(n, \varepsilon)$ or $\alpha\left(v_{1}, v_{2}\right)>\pi-\alpha_{0}(n, \varepsilon)$, where $\alpha\left(v_{1}, v_{2}\right)$ is the angle between $v_{1}$ and $v_{2}$.

(iii) $\sup \left\{d\left(q^{\prime}, q\right) \mid q^{\prime} \in M-B(r, p)\right\} \geq(\pi / 2)-c_{2}(n, \varepsilon, r)$.

(iv) $\lim _{\varepsilon \rightarrow 0} c_{1}(n, \varepsilon)=\lim _{\varepsilon \rightarrow 0} c_{2}(n, \varepsilon, r)=\lim _{\varepsilon \rightarrow 0} \alpha_{0}(n, \varepsilon)=0$. 
REMARK. One can replace the condition $K(M, g) \geq 1$ by $K(M, g) \geq C$, for any $C \in \mathbf{R}$ in Theorem 2 part (a), to estimate

$$
\operatorname{vol}_{n}\left(B\left(r^{\prime}, q\right)-B(r, p)\right) \leq \lambda_{C}\left(L(q, p), r, d(q, p), r^{\prime}\right)
$$

where $\lambda_{C}$ is defined similarly in the simplyconnected space form of constant sectional curvature $C$, instead of $S^{n}$. The analogue of Proposition 3.3 is still valid, however part (b) of Theorem 2 has no analogue for $C \leq 0$. Proofs of these are basically the same as $3.2,3,4$ with minor adjustments.

2. Notation and basic definitions. For any metric space $(X, d)$ and any subset $A$ and $r>0$ one defines $N(r, A)$ to be $\{x \in X \mid d(x, A)<r\}$, and $\bar{N}(r, A)$ be its closure and $N$ is replaced by $B$ if $A$ is a point.

$d$ represents the distance function of $(M, g)$ and $d(M, g)$ is the diameter of $(M, g)$. For $v, w$ in $U M_{p}$, the unit sphere bundle of $(M, g)$ at $p$ in $M$ one has $\alpha(v, w)$ to be the angle between them. All geodesics are parametrized by the arclength. A minimal geodesic $\gamma$ from $p$ to $q$ is $m g(p, q)$ and $\gamma(0)=p . \quad L(p, q)=\left\{\gamma^{\prime}(0) \mid \gamma\right.$ is $m g(p, q)\}$ is the link from $p$ to $q$ for any $p, q$ in $M$.

$S^{k}$ represents the unit standard sphere with its canonical metric. Let $v_{n}(r)=$ $\operatorname{vol}_{n}\left(B\left(r, p^{\prime} ; S^{n}\right)\right)$ and $v_{n}=v_{n}(\pi)=2 V_{0}$. A subset $A$ of $S^{k}$ spans $S^{k}$ if $\bar{N}(\pi / 2, A)=$ $S^{k}$ and spans strictly if $N(\pi / 2, A)=S^{k} . q$ is a nontrivial critical point for $d(p$,$) in$ the sense of Grove and Shiohama [GS] and Gromov [Gv2], if $L(q, p)$ spans $U M_{q}$.

\section{Main construction and the proof of Theorem 2.}

LEMMA 3.1. Let $A$ be closed and span $S^{m}$. Then there exists a finite subset $A^{\prime}$ of $A$, which spans $S^{m}$.

Proof. Any $B$ which spans a lower dimensional great sphere $S^{k}$ spans $S^{m}$, since for any $p$ in $S^{m}$ take $q$ to be a closest point on $S^{k}$ to $p, b$ in $B$ with $d(b, q) \leq \pi / 2$, then $d(p, q) \leq \pi / 2$ and $m g(p, q)$ is normal to $S^{k}$ and hence $d(b, p) \leq \pi / 2$. Let $B$ be closed and span $S^{k}$. If $B$ spans $S^{k}$ strictly then there is a finite subcover of $\{N(\pi / 2, b) \mid b \in$ $B\}$ to give a finite subset $B_{1}$ to span $S^{k}$. If $B$ does not span $S^{k}$ strictly, then one constructs $B_{2}$ as follows. Let $q$ be in $S^{k}-N(\pi / 2, B) . N(\pi / 2, q) \cap B=\varnothing$. Let $S_{q}^{k-1}=\left\{x \in S^{k} \mid d(x, q)=\pi / 2\right\}$ and $B_{2}=B \cap S_{q}^{k-1}$. Given any $q^{\prime}$ in $S_{q}^{k-1}$, let $\gamma$ be $m g\left(q, q^{\prime}\right)$ and $q_{n}=\gamma(1 / n), \forall n \in \mathbf{N}^{+}$. There is $p_{n}$ in $B$ with $d\left(p_{n}, q_{n}\right) \leq$ $\pi / 2, \forall n \in \mathbf{N}^{+} . \quad p_{n} \in \bar{N}\left(\pi / 2, q_{n}\right) \cap \bar{N}(\pi / 2,-q) \subseteq \bar{N}\left(1 / n, \bar{N}\left(\pi / 2, q^{\prime}\right) \cap S_{q}^{k-1}\right)$. $\bar{B} \cap \bar{N}\left(\pi / 2, q^{\prime}\right) \cap S_{q}^{k-1}=B_{2} \cap \bar{N}\left(\pi / 2, q^{\prime}\right) \neq \varnothing . B_{2}$ spans $S_{q}^{k-1}$ and hence $S^{k}$. So one obtains spanning subsets $B_{1}$ or $B_{2}$ where $B_{1}$ is finite and $B_{2}$ lies in a lower dimensional great sphere. The rest of the proof follows by starting $B=A$ and $k=m$ and reducing $k$, with the fact that $S^{0}$ is a pair of antipodal points.

3.2. Let $p^{\prime} \in S^{n}, A \subseteq U S_{p^{\prime}}^{n}$ (which is identified with $S^{n-1}$ isometrically) and $0 \leq x \leq y \leq \pi, 0 \leq z \leq \pi$ be given. Define $W(A) \subseteq S^{n}$ by $W(A)=\bar{B}\left(z, p^{\prime}\right)-$ $\bigcup_{v \in A} B\left(x, \exp _{p^{\prime}} y v\right)$ and $\lambda(A, x, y, z)=\operatorname{vol}_{n} W(A)$. Obviously if $A \subseteq B$, then $\lambda(A, x, y, z) \geq \lambda(B, x, y, z)$. For a fixed $A, \lambda$ is continuous in $x, y$, and $z$. If one fixes the number of vectors in $A$ as a finite number and varies the vectors continuously then $\lambda$ varies continuously. Clearly one can find $Y(A) \subseteq \bar{B}(\pi, 0) \subseteq T S_{p^{\prime}}^{n}$ with $\exp _{p^{\prime}} Y(A)=W(A)$. 
Proposition 3.3. Let $A$ span $S^{n-1}$. Then $\lambda(A, x, y, z) \leq \lambda\left(S^{0}, x, y, z\right)$. If $\lambda\left(S^{0}, x, y, z\right)>0$ and $x>0$, then equality holds only if $A=S^{0}$, where $S^{0}$ is an antipodal pair.

ProOF. We first prove this for finite $A=\left\{a_{1}, a_{2}, \ldots, a_{m}\right\}$ where $a_{i}$ are distinct. Let $x, y, z$ be fixed, and $\lambda(A)$ denote $\lambda(A, x, y, z)$. Given $0 \leq \beta \leq \pi$, take $v, w \in U S_{p^{\prime}}^{n}$ with $\alpha(v, w)=\beta$, and define $I(\beta)$ to be the largest subset of $[0, z]$ with $\left(\exp _{p^{\prime}} I(\beta) v\right) \cap B\left(x, \exp _{p^{\prime}} y w\right)=\varnothing$. I( $\left.\beta\right) \subseteq I\left(\beta^{\prime}\right)$ if $\beta \leq \beta^{\prime}$. Let $f(\beta)=\int_{I(\beta)} \sin ^{n-1} t d t$. Then

$$
\lambda(A)=\int_{v \in U S^{n}\left(p^{\prime}\right)} \int_{I(\alpha(v, A))} \sin ^{n-1} t d t d \nu=\int_{v \in S^{n-1}} f(\alpha(v, A)) d \nu,
$$

where $d \nu$ is the volume form of $S^{n-1}$ and $\alpha(v, A)=\inf \{\alpha(v, w) \mid w \in A\}$. Let $\omega(r)=\operatorname{vol}_{n-2}\left(\partial B\left(r\right.\right.$, point; $\left.\left.S^{n-1}\right)\right)$ and $\Phi(r, A)=(\omega(r))^{-1} \operatorname{vol}_{n-2}(\partial B(r, A))$. $\lim _{r \rightarrow 0} \Phi(r, A)=m$, and in fact $\Phi$ is continuous for $r<\pi / 2$.

$$
\Phi(r, A) \omega(\pi / 2)=\sum_{i=1, \ldots, m} \operatorname{vol}_{n-2}\left\{v \in U S_{a_{i}}^{n-1} \mid d\left(\exp _{a_{i}}(r v), A\right)=r\right\} \quad \text { if } r<\pi / 2 .
$$

Hence $\Phi(r, A) \leq \Phi\left(r^{\prime}, A\right)$ if $r>r^{\prime}$. By the coarea formula [Ch], $f$ being constant on the level sets of the distance function from $A$, whose gradient is defined and has length 1 on an open set that has complement with measure 0 , one has

$$
\int_{[0, \pi / 2]} \Phi(r, A) \omega(r) d r=v_{n-1}, \quad \text { and } \quad \int_{[0, \pi / 2]} \Phi(r, A) \omega(r) f(r) d r=\lambda(A)
$$

Define $\mu(t, A)=\int_{[0, t]} \Phi(r, A) \omega(r) d r=\operatorname{vol}_{n-1} N(t, A)$.

ClaIM. $\mu(t, A) \geq \mu\left(t, S^{0}\right)$ for all $t \in[0, \pi / 2]$. $m \geq 2$, and $m=2$ only if $A=S^{0}$, since $A$ spans $S^{n-1}$. So, we may assume that $m \geq 3$. Let $h(t)=\mu(t, A)-\mu\left(t, S^{0}\right)$ and $u(r)=\Phi(r, A)-\Phi\left(r, S^{0}\right)=\Phi(r, A)-2$, so that $\int_{[0, t]} u(r) \omega(r) d r=h(t)$. $h(0)=h(\pi / 2)=0, u(r)$ is decreasing and $\omega(r)>0$, if $r>0$. For small $r, u(r)=$ $m-2>0$, and $h(t)>0$ for small $t>0$. Let $\pi / 2 \geq a>0$ be the smallest with $h(a)=0 . u(a)<0$, and if $a$ were less than $\pi / 2$ then $h(\pi / 2)$ would be negative. So $a=\pi / 2$ and $h \geq 0$.

Since $f$ is increasing,

$$
\begin{aligned}
\operatorname{vol}_{n-1}\{v \mid f(\alpha(v, A)) \leq f(t)\} & =\mu\left(t^{\prime}, A\right) \geq \mu\left(t^{\prime}, S^{0}\right) \\
& =\operatorname{vol}_{n-1}\left\{v \mid f\left(\alpha\left(v, S^{0}\right)\right) \leq f(t)\right\}
\end{aligned}
$$

where $t^{\prime}=\sup \left\{t^{\prime \prime} \mid f\left(t^{\prime \prime}\right)=f(t)\right\}$. The inequality of the proposition follows the formula $\int_{v \in S^{n-1}} f(\alpha(v, A)) d \nu=\lambda(A)$. In the case of equality, since $x>0$ and $\lambda\left(S^{0}\right)>0, f$ is strictly increasing and positive in a neighborhood of 0 . For small $t, \mu(t, A)=\mu\left(t, S^{0}\right)$ and hence $\Phi(r, A)=\Phi\left(r, S^{0}\right)=2$ for small $r$. Since $A$ spans $S^{n-1}, A=S^{0}$. In the case of $A$ not being finite, one finds a finite subset $A^{\prime}$ of $A$, which spans $S^{n-1}$ by Lemma 3.1 and $\lambda(A) \leq \lambda\left(A^{\prime}\right) \leq \lambda\left(S^{0}\right)$, and the equality never occurs since $\bar{A}=\bar{A}^{\prime}$ is necessary for $\lambda(A)=\lambda\left(A^{\prime}\right)$ with $A^{\prime} \subseteq A$.

3.4. Let $(M, g)$ be with $K(M, g) \geq 1, d(M, g)=z, p, q \in M$ with $d(p, q)=y$ and $x=r \leq d(p, q)$ be arbitrary. Let $T S_{p^{\prime}}^{n}$ and $T M_{q}$ be identified naturally by an isometry. Let $E_{q}$ be the closed subset of $T M_{q}$ bounded by the tangential cutlocus of $q$. Obviously $\exp _{q} E_{q}=M$. Let $D_{q}=E_{q} \cap Y(L(q, p))$, for $x, y$, and $z$ as above, 
see 3.2. Let $q^{\prime} \in M-B(r, p)$ and $w^{\prime} \in E_{q}$ with $\exp _{q} w^{\prime}=q^{\prime}$. By Toponogov's Theorem [CE], for any $w \in L(q, p), d\left(\exp _{p^{\prime}} y w, \exp _{p^{\prime}} w^{\prime}\right) \geq d\left(\exp _{q} y w, \exp _{q} w^{\prime}\right)=$ $d\left(p, q^{\prime}\right) \geq r$. So, $w^{\prime} \in D_{q}$ and hence $M-B(r, p) \subseteq \exp _{q} D_{q}$. Even though $D_{q}$ is not star shaped, the comparison of the pull-back volume form to the standard one obtained from $S^{n}$ is valid on $D_{q}[\mathrm{BC}]$.

$$
\begin{aligned}
\operatorname{vol}_{n}(M-B(r, p)) & \leq \operatorname{vol}_{n}\left(\exp _{q} D_{q}\right) \leq \operatorname{vol}_{n}\left(\exp _{p^{\prime}} D_{q}\right) \leq \operatorname{vol}_{n}\left(\exp _{p^{\prime}} Y(L(q, p))\right) \\
& =\lambda(L(q, p), r, d(p, q), d(M, g)) .
\end{aligned}
$$

$v_{n}(r)+\lambda(L(q, p), r, d(p, q), d(M, g)) \geq v(M, g)$.

3.5. Let $(M, g)$ be a Riemannian manifold with $K(M, g) \geq 1, v(M, g)>V_{0}-\varepsilon$, $d(M, g) \leq \pi / 2, p \in M$ and $q$ be a nontrivial critical point for $d(p$,$) .$

3.6. Given $a, b$, and $c$, define $\tau(a, b, c)=\operatorname{vol}_{n}\left(B\left(a, p_{0}\right) \cap B\left(b, p_{1}\right)\right)$ where $p_{0}, p_{1} \in$ $S^{n}$ with $d\left(p_{0}, p_{1}\right)=c$.

LEMMA 3.7. If 3.5 holds, then $d(p, q)>(\pi / 2)-c_{1}(n, \varepsilon)$, for some $c_{1}>0$.

Proof. Let $d(p, q)=r$ and $A=L(p, q)$. Take $Y(A)$ as in 3.2 with $x=z=\pi / 2$, $y=r+(\pi / 2)$, and identify $T M_{p}$ and $T S_{p^{\prime}}^{n}$ by an isometry.

ClaIM. $\exp _{p} Y(A)=M$. Proof by contradiction. Suppose there exists $q^{\prime} \in M-$ $\exp _{p} Y(A)$, and let $a=d\left(q^{\prime}, p\right), v \in L\left(p, q^{\prime}\right)$. $a v \in \bar{B}(\pi / 2,0)-Y(A) \subseteq T M_{p}$. There exists $w \in A$ with $\alpha(w, v) \leq \pi / 2, a v \in \bar{B}(\pi / 2,0)-Y(\{w\}) . d\left(\exp _{p^{\prime}} r w, \exp _{p^{\prime}} a v\right)=$ $b \leq \pi / 2$ in $S^{n}$. Let $\beta(r, b, a)$ be the angle across the side of length $a$ in a geodesic triangle of side lengths $r, a, b$ on $S^{2}$. By Toponogov's Theorem [CE], $d\left(q, q^{\prime}\right)=b^{\prime} \leq$ $b$ and $\alpha\left(L(q, p), L\left(q, q^{\prime}\right)\right) \geq \beta\left(r, b^{\prime}, a\right) \geq \beta(r, b, a)>\pi / 2$ which is not the case since $q$ is a nontrivial critical point for $d(p$,$) . So the claim holds.$

$v(M, g) \leq \lambda(A, \pi / 2, r+(\pi / 2), \pi / 2) \leq \lambda\left(\left\{v_{0}\right\}, \pi / 2, r+(\pi / 2), \pi / 2\right)$. Hence one chooses $c_{1}(\varepsilon, n)$ with $\varepsilon=\tau\left(\pi / 2, \pi / 2, \pi-c_{1}(\varepsilon, n)\right)$ to prove the lemma.

LEMMA 3.8. If 3.5 holds, then for all $v, w$ in $L(q, p)$,

$$
\alpha(v, w) \in\left[0, \alpha_{0}\right) \cup\left(\pi-\alpha_{0}, \pi\right]
$$

for some $\alpha_{0}=\alpha_{0}(\varepsilon, n)>0$.

ProOF. Suppose that there exist $v$ and $w$ in $L(q, p)$ with $\theta \leq \alpha(v, w) \leq \pi-\theta$ for some $\theta>0$. Then there exists $w^{\prime}$ in $L(q, p)$ with $\alpha\left(v, w^{\prime}\right)$ and $\alpha\left(w, w^{\prime}\right) \geq \theta / 2$. Let $a=\sin \left((\pi / 2)-c_{1}(\varepsilon, n)\right)$.

$$
\begin{aligned}
v(M, g) & \leq v_{n}(\theta a / 4)+\lambda(L(q, p), \theta a / 4, d(p, q), d(M, g)) \\
& \leq v_{n}(\theta a / 4)+\lambda\left(\left\{v, w, w^{\prime}\right\}, \theta a / 4, \pi / 2, \pi / 2\right) \\
& \leq \lambda(\{v\}, \theta a / 4, \pi / 2, \pi / 2) .
\end{aligned}
$$

So one chooses $\alpha_{0}(\varepsilon, n)$ with $\varepsilon=\tau\left(\pi / 2, a \alpha_{0}(\varepsilon, n) / 4, \pi / 2\right)$ to prove the lemma.

LEMMA 3.9. If 3.5 holds, then $\sup \left\{d\left(q^{\prime}, q\right) \mid q^{\prime} \in M-B(r, p)\right\} \geq \pi / 2-c_{2}(\varepsilon, r, n)$, for some $c_{2}>0$ and $r \leq d(p, q)$.

PROOF. Let $z=\sup \left\{d\left(q^{\prime}, q\right) \mid q^{\prime} \in M-B(r, p)\right\}$.

$$
\begin{aligned}
v(M, g) & \leq \lambda(L(q, p), r, d(p, q), z)+v_{n}(r) \leq \lambda\left(S^{0}, r, \pi / 2, z\right)+v_{n}(r) \\
& =v_{n}(z)+v_{n}(r)-2 \tau(r, z, \pi / 2)=V_{0}-c(r, z, n) .
\end{aligned}
$$

So one chooses $c_{2}(\varepsilon, r, n)$ appropriately to prove the lemma.

ProOF OF THEOREM 2. This follows 3.2, Proposition 3.3, 3.4, Lemmas 3.7, 3.8 , and 3.9 immediately. 


\section{Proof of Theorem 1.}

4.1. Let $\left(M, g_{k}\right)$ be a sequence of smooth Riemannian metrics with $1 \leq K\left(M, g_{k}\right)$ $\leq K, d\left(M, g_{k}\right)$ converging to $\pi / 2$ and $v\left(M, g_{k}\right)$ converging to $V_{0}$. By Gromov's Compactness Theorem $[\mathbf{G v}, \mathbf{P}]$, one can extract a subsequence of $\left(M, g_{m}\right)$ converging to $\left(M, g_{0}\right)$ in the Hausdorff-Lipschitz sense, where $g_{0}$ is $C^{\mathbf{1}, \alpha}, \alpha<1 . d\left(M, g_{0}\right)=\pi / 2$ and $v\left(M, g_{0}\right)=V_{0}$. One defines $d_{i}(p, q)=d\left(p, q ; g_{i}\right)$ for $i=0$ or $m$, and the critical points of $d_{0}(p$,$) as in \S 2$.

PROPOSITION 4.2. Let $p \in\left(M, g_{0}\right)$ and $q$ be a nontrivial critical point for $d_{0}(p$,$) . Then,$

(a) $d_{0}(p, q)=\pi / 2$,

(b) there exists $p_{0} \in M$ such that $d_{0}\left(p_{0}, p\right)=d_{0}\left(p_{0}, q\right)=\pi / 2$, and

(c) $L\left(q, p ; g_{0}\right)$ is an antipodal pair.

ProOF. Let $A=\left\{a_{1}, a_{2}, \ldots, a_{k}\right\}$ be a finite spanning subset of $L\left(q, p ; g_{0}\right)$ by Lemma 3.1. Let $x$ be fixed with $0<x \leq d_{0}(p, q)$. Define $C_{0}(x) \geq 0$ by

$$
\max \left\{d_{0}\left(q^{\prime}, q\right) \mid q^{\prime} \in M-B(x, p)\right\}=(\pi / 2)-C_{0}(x) .
$$

Given $\varepsilon>0$ arbitrarily small, choose $m \in \mathbf{N}^{+}$sufficiently large such that

1. $d_{m}\left(q_{i}, p\right)<\varepsilon$, where $q_{i}=\exp \left(q ; g_{m}\right)\left(d_{0}(p, q) a_{i} /\left\|a_{i}\right\|_{m}\right)$, for $1 \leq i \leq k$,

2. $d\left(M, g_{m}\right) \leq(\pi / 2)+\varepsilon$, and

3. $\max \left\{d_{m}\left(q^{\prime}, q\right) \mid q^{\prime} \in M-B\left(x, p ; g_{m}\right)\right\} \leq(\pi / 2)-C_{0}(x)+\varepsilon$.

For $0<x \leq y=d_{0}(p, q)$ and $z=(\pi / 2)+\varepsilon$, construct $Y\left(\psi_{m}(A)\right)$ as in 3.4, where $\psi_{m}: U M\left(g_{m}\right)_{q} \rightarrow U S_{p^{\prime}}^{n}$ is an isometry. By Toponogov's Theorem as in 3.4

$$
\begin{gathered}
M-\exp \left(q ; g_{m}\right) \psi_{m}^{-1} Y\left(\psi_{m}(A)\right) \subseteq \bigcup_{i=1, \ldots, k} B\left(x, q_{i} ; g_{m}\right) \subseteq B\left(x+\varepsilon, p, g_{m}\right), \\
M-B\left(x+\varepsilon, p, g_{m}\right) \subseteq \exp \left(q ; g_{m}\right)\left(\psi_{m}^{-1}\left(Y\left(\psi_{m}(A)\right) \cap \bar{B}\left((\pi / 2)-C_{0}(x)+\varepsilon, 0\right)\right)\right), \\
v\left(M, g_{m}\right) \leq v_{n}(x+\varepsilon)+\lambda\left(\psi_{m}(A), x, d_{0}(p, q),(\pi / 2)-C_{0}(x)+\varepsilon\right) .
\end{gathered}
$$

Let $\varepsilon \rightarrow 0$, choose $\psi_{m}$ so that $\psi_{m} \rightarrow \psi_{0}$, where $\psi_{0}: U M\left(g_{0}\right)_{q} \rightarrow U S_{p^{\prime}}^{n}$ is an isometry, by extracting a subsequence if necessary.

$$
\begin{aligned}
V_{0}=v\left(M, g_{0}\right) & \leq v_{n}(x)+\lambda\left(\psi_{0}(A), x, d_{0}(p, q),(\pi / 2)-C_{0}(x)\right) \\
& \leq v_{n}(x)+\lambda\left(S^{0}, x, d_{0}(p, q),(\pi / 2)-C_{0}(x)\right) .
\end{aligned}
$$

If $C_{0}(x)>0$ or $d_{0}(p, q)<\pi / 2$, then $v_{n}(x)+\lambda\left(S^{0}, x, d_{0}(p, q),(\pi / 2)-C_{0}(x)\right)<V_{0}$. Hence, $C_{0}(x)=0, d_{0}(p, q)=\pi / 2$, and $\psi_{0}(A)=S^{0}$, by Proposition 3.3.

The rest of the proof of Theorem 1 is based on Proposition 4.2 and a sequence of results proved formally in [D] as follows. Let $p \in\left(M, g_{0}\right)$ be fixed, $C=$ $\left\{q \in\left(M, g_{0}\right) \mid d_{0}(p, q)=\pi / 2\right\}$, and $C^{\prime}=\left\{q \in\left(M, g_{0}\right) \mid d_{0}(q, C)=\pi / 2\right\} . C$ and $C^{\prime}$ are convex, that is any minimal geodesic joining two points of the set lies in the set, by Toponogov's Theorem (see $[\mathbf{D}, 4.5]$ ). So both are totally geodesic submanifolds of $\left(M, g_{0}\right)$ possibly with boundary. $\partial C^{\prime} \neq \varnothing$, since otherwise the furthest point on $C^{\prime}$ from $p$ would be a critical point for $d_{0}(p$,$) and by Proposition 4.2, it$ would be in $C$. Suppose that $\partial C \neq \varnothing$, and take $p_{0}$ to be the furthest point in $C$ from $\partial C$, see $\left[\mathbf{D}, 4.6 .2\right.$ and 6.9], and $p_{1} \in C$ with $d_{0}\left(p_{0}, p_{1}\right)=\pi / 2$, by 4.2.b. By Proposition 4.2.c, $p_{0}$ and $p_{1}$ lie on a closed geodesic $\gamma$ which lies in $C . \gamma \cap \partial C=\varnothing$, since any closed geodesic in a convex set intersecting with the boundary lies on the 
boundary and $p_{0} \in \operatorname{int}(C)$. On the other hand $d(, \partial C)$ cannot have any local minimum on geodesic segments lying in $\operatorname{int}(C)$, since $K\left(M, g_{m}\right) \geq 1$, see Lemma 6.8 in [D]. Consequently, $\partial C=\varnothing \cdot d_{0}(p$,$) has no critical points on M-C$, therefore there exists sufficiently large $m$ such that $d_{m}(p$,$) has no critical points on M-N(\delta, C)$, for small $\delta>0$. By using the techniques of [GS] one can show that $M-N(\delta, C)$ is homeomorphic to an $n$-ball, and the unit normal bundle UNC of $C$ in $M$ with respect to $g_{0}$ is homeomorphic to $S^{n-1} . E=\bigcup_{q \in C} L\left(q, p ; g_{0}\right)$ is a subbundle of UNC. $E$ has to be UNC, since UNC-\{point\} is contractible and $\mathbf{Z}_{2} \rightarrow E \rightarrow C$ cannot be null-homotopic, [GG3]. Hence $\partial(M-N(\delta, C))=S^{n-1} \rightarrow C$ is a double covering and $M$ has the homotopy type of $\mathbf{R P}^{n}$. By Theorem 6.22 of $[\mathbf{D}],\left(M, g_{0}\right)$ is isometric to $\mathbf{R P}^{n}$.

The proof of the Corollary of Theorem 1 follows by recalling the Generalized Sphere Theorem [GS], see $\S 1$, and the Finiteness Theorem of Cheeger $[\mathbf{C}]$, that there are finitely many diffeomorphism types of Riemannian manifolds with $1 \leq$ $K(M, g) \leq K, v(M, g)>V, d(M, g) \leq \pi$ and hence inj-rad $(M, g)>C(K, V, \pi)$.

\section{REFERENCES}

[BC] R. L. Bishop and R. J. Crittenden, Geometry of manifolds, Pure and Appl. Math., vol. 15, Academic Press, New York and London, 1964.

[Ch] I. Chavel, Eigenvalues in Riemannian geometry, Pure and Appl. Math., vol. 115, Academic Press, Orlando, 1984.

[C] J. Cheeger, Finiteness theorems for Riemannian manifolds, Amer. J. Math. 92 (1970), 61-74.

[CE] J. Cheeger and D. G. Ebin, Comparison theorems in Riemannian geometry, North-Holland, Amsterdam, 1975.

[D] O. C. Durumeric, A generalization of Berger's theorem on almost 1/4-pinched manifolds. II, J. Differential Geom. 26 (1987), 101-139.

[GG1] D. Gromoll and K. Grove, Rigidity of positively curved manifolds with large diameter, Ann. of Math. Studies, no. 102, Princeton Univ. Press, Princeton, N.J., 1981, pp. 203-207.

[GG2] _ One dimensional metric foliations in constant curvature spaces, Differential Geometry and Complex Analysis, a volume dedicated to H. E. Rauch (I. Chavel and H. M. Farkas, eds.), Springer-Verlag, Berlin and New York, 1985.

[GG3] _ A generalization of Berger's rigidity theorem for positively curved manifolds, Ann. Sci. Ecole Norm. Sup. (4) 20 (1987), 227-239.

[GG4] _ On the low dimensional metric foliations of Euclidean spheres, J. Differential Geometry (to appear).

[Gv] M. Gromov, Structures metriques pour les varietes riemanniennes (J. Lafontaine and P. Pansu, eds.), Cedic/Ferdnan Nathan, Paris, 1981.

[Gv2] _ Curvature, diameter and Betti numbers, Comment. Math. Helv. 56 (1981), 179-195.

[GP1] K. Grove and P. Petersen, Bounding homotopy types by geometry, Ann. of Math. (2) 128 (1988), 195-206.

[GP2] — Homotopy types of positively curved manifolds with large volume, Amer. J. Math. (to appear).

[GS] K. Grove and K. Shiohama, A generalized sphere theorem, Ann. of Math. (2) 106 (1977), 201-211.

[P] S. Peters, Convergence of Riemannian manifolds, Compositio Math. 62 (1987), 3-16.

\section{Department of Mathematics, Princeton Univeristy, Princeton, New Jersey 08544}

Current address: Department of Mathematics, University of Iowa, Iowa City, Iowa 52242 